\title{
Controlling the surface structure of electrospun fibers: Effect on endothelial cells and blood coagulation
}

\author{
Anne-Sophie Mertgen, ${ }^{1,2, a)}$ Gökçe Yazgan, ${ }^{1,2, a)}$ Anne Géraldine Guex, ${ }^{1,2, b)}$ \\ Giuseppino Fortunato, ${ }^{2}$ Eike Müller, ${ }^{1}$ Lukas Huber, ${ }^{3}$ René Schneider, ${ }^{4}$ Marzia Brunelli, ${ }^{2}$ \\ René M. Rossi, ${ }^{2}$ Katharina Maniura-Weber, ${ }^{1}$ and Markus Rottmar ${ }^{1, b)}$ \\ ${ }^{1}$ Empa, Swiss Federal Laboratories for Materials Science and Technology, Biointerfaces, 9014 St. Gallen, \\ Switzerland \\ ${ }^{2}$ Laboratory for Biomimetic Membranes and Textiles, Empa, Swiss Federal Laboratories for Materials Science \\ and Technology, St. Gallen 9014, Switzerland \\ ${ }^{3}$ Empa, Swiss Federal Laboratories for Materials Science and Technology, Building Energy Materials and \\ Components, 8600 Dübendorf, Switzerland \\ ${ }^{4}$ Empa, Swiss Federal Laboratories for Materials Science and Technology, Functional Polymers, 8600 \\ Dübendorf, Switzerland
}

(Received 9 July 2018; accepted 30 August 2018; published 24 September 2018)

\begin{abstract}
The influence of nano- or micron-sized structures on polymer films as well as the impact of fiber diameter of electrospun membranes on endothelial cell (EC) and blood response has been studied for vascular tissue engineering applications. However, the influence of surface structures on micronsized fibers on endothelial cells and blood interaction is currently not known. In this work, electrospun membranes with distinct fiber surface structures were designed to study their influence on the endothelial cell viability and thrombogenicity. The thermodynamically derived Hansen-solubilityparameters model accurately predicted the formation of solvent dependent fiber surface structured poly(caprolactone) membranes. The electrospun membranes composed of microfibers (MF) or structured MF were of similar fiber diameter, macroscopic roughness, wettability, and elastic modulus. In vitro evaluation with ECs demonstrated that cell proliferation and morphology were not affected by the fiber surface structure. Similarly, investigating the blood response to the fiber meshes showed comparable fibrin network formation and platelet activation on MF and structured MF. Even though the presented results provide evidence that surface structures on MF appear neither to affect EC viability nor blood coagulation, they shed light on the complexity and challenges when studying biology-material interactions. They thereby contribute to the understanding of EC and blood-material interaction on electrospun membranes. Published by the AVS.

https://doi.org/10.1116/1.5047668
\end{abstract}

\section{INTRODUCTION}

Cardiovascular diseases, such as atherosclerosis and its progressive form vascular stenosis, are the major cause of mortality worldwide. ${ }^{1}$ Cardiac and peripheral bypass surgery, in which the blocked part of the blood vessel is replaced by a vascular graft, is the standard treatment method for such diseases. While autologous grafts (i.e., saphenous veins) are still outperforming their synthetic substitutes, they have limited availability and can cause donor site morbidity. ${ }^{2}$ Commercially available synthetic vascular grafts are mainly made of nondegradable expanded poly(tetrafluoro ethylene) (PTFE) or poly(ethylene terephthalate). Although they perform with good patency rates for large diameter grafts, they suffer from restenosis in small diameter vascular grafts with a diameter of $<6 \mathrm{~mm}$ mostly due to thrombosis and a mismatch of compliance. ${ }^{2,3}$

As an alternative approach, vascular tissue engineering aims to create a functional endothelium on the luminal surface of the graft that mimics the natural blood-tissue

a) A.-S. Mertgen and G. Yazgan contributed equally to this work.

b) Authors to whom correspondence should be addressed: markus.rottmar@ empa.ch and geraldine.guex@empa.ch interface to avoid coagulation. Scaffolds for such tissue engineered vascular grafts need to exhibit a variety of characteristics including mechanical properties similar to those of blood vessels, topological mimicry of the native cellular microenvironment, and controlled biodegradability for gradual replacement by regenerated natural tissue. In case of local delamination of the endothelium, they additionally have to provide low thrombogenicity. ${ }^{4,5}$

In order to enhance endothelialization of in vitro engineered vascular grafts, tailoring surface properties and in particular topography adaption have a pivotal role. It has been shown that different surface structures and patterns alter endothelial cell (EC) behavior. For instance, substrates with ridge and groove widths of $1 \mu \mathrm{m}$ and ridge heights of 100 , 400 , or $1000 \mathrm{~nm}$ can modulate EC migration behavior and adhesion force. $^{6} 2 \mathrm{D}$ substrates with pores in the range of $1-1.5 \mu \mathrm{m}$ in diameter enhanced aortic EC attachment and proliferation compared to substrates with pores smaller than $15 \mathrm{~nm}$ in diameter. ${ }^{7}$ Additionally, lowered thrombogenicity can also be achieved by implementing a surface topography in the submicron range to decrease the adhesion and activation of platelets. ${ }^{8-10}$ For instance, in the study of Koh et al., the lowest amount of fibrinogen adsorption and platelet activation 
was observed on poly(lactic-co-glycolic acid) (PLGA) submicron range pillared surfaces with high aspect ratio and reduced interspacing $(<200 \mathrm{~nm})$ compared to unstructured PLGA films. ${ }^{8}$

Creating micro- or nano-fibrous scaffolds by electrospinning has found widespread interest in research to architecturally mimic the fibrous structure of native extracellular matrix. A key benefit of this technique is the freedom in material choice, as many degradable or stable polymers that offer excellent cytocompatibility can be processed by electrospinning. ${ }^{4}$ Importantly, fiber diameter and 3D scaffold architecture can be controlled by tuning the spinning parameters. Varying the topographies of electrospun membranes caused by different fiber diameters were previously shown to induce altered EC fate as well as platelet activation and blood coagulation. ${ }^{11,12}$ It has been demonstrated in vitro that scaffolds with micronsized fibers can support human umbilical vein endothelial cell (HUVEC) monolayer formation. ${ }^{12}$ In contrast, nanosized fibers induced lower platelet adhesion and activation and thus reduced coagulation after blood incubation, when compared to micron-sized fibers. For instance, Milleret et al. ${ }^{11}$ reported increasing platelet adhesion and activation on electrospun fibers of Degrapol ${ }^{\circledR}$ or PLGA with increasing diameters, similar to the trend reported with nondegradable polymers, namely, poly(vinylidene fluoride-co-hexafluoropropylene) or poly(tetrafluoro ethylene). ${ }^{13,14}$

Besides fiber diameter and related interfiber porosity, surface structures on individual fibers can also steer cellular behavior. The formation of such structures is driven by polymer phase separation of solvent/nonsolvent mixtures or the interaction with water vapor from the air during the drawing and evaporation phase in the electrospinning process. The creation of such topographical cues can thus be steered by carefully choosing solvent mixtures and/or relative environmental humidity settings during the processing. ${ }^{15,16}$

The influence of such fiber surface structures has been reported by Zhou et al. They showed that nanosized structures on micron-sized poly(-L-lactic acid) (PLLA) fibers adsorb more proteins due to their increased surface area and hydrophobicity and thus enhance proliferation of smooth muscle cells (SMCs). ${ }^{15}$

Although electrospun scaffolds with micron-sized fibers have demonstrated endothelialization potential and elastic behavior in vivo, they are more prone to elicit thrombogenic events. ${ }^{11,17,18}$ Interestingly, structures generated on flat surfaces have the capacity not only to induce better adhesion of EC, but also to improve hemocompatibility of a material. ${ }^{6,8}$ Reduced blood coagulation is potentially caused by surface structures reducing the contact between platelets and surface as hypothesized by Hulander et al. ${ }^{9}$ This suggests that nanosized structures on micron-sized fibers can enhance EC attachment and reduce thrombogenicity of micron-sized fibers upon blood contact due to decreased surface contact.

In this study, the behavior of HUVECs as well as platelet activation and blood coagulation on electrospun membranes with distinct surface structures was studied. Electrospun poly(caprolactone) (PCL) membranes were developed with comparable fiber diameters but with two distinct fiber surface structures by the use of a prediction tool utilizing Hansen-solubility-parameters (HSP). ${ }^{16}$ PCL was chosen as the substrate material, because it is biodegradable, possesses favorable mechanical properties for vascular applications, can easily be processed by electrospinning, and is widely used in vascular tissue engineering research. ${ }^{19}$

\section{EXPERIMENT}

\section{A. Hansen-solubility-parameters}

HSP were used to assess the solubility of PCL in chloroform $\left(\mathrm{CHCl}_{3}\right)$, dimethylformamide (DMF), dimethyl sulfoxide (DMSO), acetonitrile, or methanol as detailed in previous work. ${ }^{16}$ Briefly, HSP construct a 3D Hansen space with its coordinates being dispersive interactions $(\delta D)$, polar interactions $(\delta P)$, and hydrogen bonding $(\delta H)$ in units of $\mathrm{MPa}^{1 / 2}$. Solvents are depicted as single points in this Hansen space, while polymers are represented as spheres with their interaction radius of $R$. $^{20}$

The interaction of PCL and solvent mixtures was evaluated based on the relative energy distance (RED) between them with respect to time [Eq. (1)]

$$
\mathrm{RED}=\mathrm{Ra} / R
$$

where $\mathrm{Ra}$ is the distance of the solvent to the center of the polymer solubility sphere [Eq. (2)]:

$$
\mathrm{Ra}=\sqrt{ }\left[4(\Delta \delta D)^{2}+(\Delta \delta P)^{2}+(\Delta \delta H)^{2}\right] .
$$

If the RED value of a polymer-solvent pair is below 1.0, the polymer can be dissolved in that solvent, whereas above 1.0, phase separation or nonsolubility is observed.

The HSP values for PCL, solvents, or solvent mixtures were obtained from HSP in Practice (HSPIP) software. ${ }^{21}$

\section{B. Solvent evaporation}

Solvent evaporation was evaluated with HSPIP software by accounting for the relative evaporation rates (RERs) and activity coefficients of the solvents. RERs were given in comparison to the evaporation of standard solvent butyl acetate, by defining that it evaporates in 100 time units. ${ }^{21}$

If air saturated with water vapor [i.e., relative humidity (RH) at $100 \%$ ] encounters a surface that is colder than its own temperature, water vapor condensation will occur. This water vapor condensation condition is given in the following equation: ${ }^{16}$

$$
\Delta T=\left(T_{\mathrm{dp}}-T_{\mathrm{wb}}\right)>0,
$$

where $T_{\mathrm{dp}}$ is the dew point (the temperature limit to which air can be cooled down reaching full water vapor saturation) and $T_{\mathrm{wb}}$ is the wet bulb temperature (the temperature on the jet surface), which was calculated iteratively. ${ }^{16}$

\section{Electrospun membrane production}

PCL $\quad\left(M_{n}=70000-90000 \mathrm{~g} \mathrm{~mol}^{-1}\right.$; Sigma-Aldrich, Switzerland) solutions were prepared at a concentration of 
$18 \%(\mathrm{w} / \mathrm{v})$ by using either mixtures of $\mathrm{CHCl}_{3}$ (ReagentPlus $\geq 99.8 \%$; Sigma-Aldrich, Switzerland): DMSO (Sigma-Aldrich, Switzerland) or $\mathrm{CHCl}_{3}: N, N$-DMF (VWR Chemicals, France) with a volume ratio of $9: 1$. All solutions were processed into fibers at $35 \% \mathrm{RH}$ and $20 \pm 0.1^{\circ} \mathrm{C}$ temperature by a custombuilt electrospinning setup as described previously. ${ }^{22}$ Flow rate was set to $30 \mu \mathrm{min}^{-1}$ for both solutions and a potential of $+10 \mathrm{kV}$ on the needle and $-5 \mathrm{kV}$ on the counter electrode with a fixed tip-to-collector distance of $25 \mathrm{~cm}$ was applied.

\section{Characterization of electrospun membranes}

Electrospun fiber diameter distribution and surface structure were investigated using the Scanning Electron Microscope (SEM) (Hitachi S-4800, Hitachi High-Technologies, Japan) with a $2 \mathrm{kV}$ accelerating voltage and a $10 \mu \mathrm{A}$ current flow. The samples were sputter coated with Gold/Palladium (80/20) (45 mA, $2 \times 10^{-5}$ mbar; Leica EM ACE 600, Austria) of $5 \mathrm{~nm}$ thickness to increase the electrical conductivity prior to examination. The mean diameters of the fibers were calculated based on the measurements of 100 individual fibers from five SEM micrographs per condition performed with IMAGE J FREE software (https://imagej.nih.gov/ij/).

Krypton adsorption and desorption isotherms at $77 \mathrm{~K}$ were collected on a Micromeritics 3flex surface area and porosity analyzer. Each type of membrane was measured three times independently by cutting approximately $100 \mathrm{mg}$ of membranes which were degassed at $50{ }^{\circ} \mathrm{C}$ for $20 \mathrm{~h}$ at a pressure of $1.3 \times 10^{-2} \mathrm{mbar}$. The total surface area was calculated by the Brunauer-Emmet-Teller (BET) method. ${ }^{23}$

To evaluate the surface morphology and roughness, a nondestructive, confocal 3D Optical Surface Metrology System (DCM8, Leica Microsystems AG, Switzerland) with a Leica EPI 50× objective was used. For each sample, three different spots with an area of $264 \mu \mathrm{m} \times 351 \mu \mathrm{m}$ were scanned and the root-mean-square roughness $\left(S_{q}\right)$ was calculated according to ISO 25178 (2012).

For static water contact angle (WCA) measurement, electrospun membranes were cut into patches of $6 \mathrm{~mm}$ in diameter. 2 $\mu \mathrm{l}$ of distilled water was applied on each patch (three patches per membrane type, three drops per membrane). The WCA on the electrospun membranes was evaluated by the use of an optical contact meter equipment (Krüss GmbH, Germany) and calculated automatically by the equipment's software.

The tensile properties of electrospun membranes were assessed using an Instron 4500 apparatus (USA), equipped with a $100 \mathrm{~N}$ load cell. Prior to mechanical testing, the thickness of the samples $(1 \times 5 \mathrm{~cm})$ was measured using a profilometer (Veeco, Dektak 150, USA) and the samples were equilibrated at $23{ }^{\circ} \mathrm{C}$ and $50 \%$ relative humidity overnight to adapt to the tensile testing environmental conditions. The elastic modulus of three individual patches per condition was evaluated by applying loading and unloading ramps at a rate of $10 \mathrm{~mm} \mathrm{~min}^{-1}$. Nominal stress was calculated by normalizing the measured load to the initial cross-sectional area of the fibrous membranes, calculated by the measured sample thickness and width.

\section{E. Protein adsorption}

Forty-eight-well cell culture plates (TPP Omnilab, Switzerland) were coated with a poly(dimethylsiloxane) (Sylgard 184, Sutter Kunststoffe, Switzerland) layer prior to fixing electrospun membranes of $6 \mathrm{~mm}$ in diameter with stainless steel minutiae insect pins (EntoSPHINX, Czech Republic). ${ }^{24}$ Membranes were prewetted in $70 \%$ ethanol solution for $3 \mathrm{~min}$ and subsequently washed in phosphate buffered saline solution (PBS, Sigma-Aldrich, Switzerland). $200 \mu \mathrm{l}$ of human fibrinogen (Sigma-Aldrich, Switzerland) at a concentration of $7 \mu \mathrm{g} \mathrm{ml}^{-1}$ in PBS was added to each well for $60 \mathrm{~min}$ before washing three times for $5 \mathrm{~min}$ with $500 \mu \mathrm{l}$ PBS. To avoid unspecific binding, membranes were blocked for $60 \mathrm{~min}$ in $4 \%(\mathrm{w} / \mathrm{v})$ bovine serum albumin (BSA; Sigma-Aldrich, Switzerland) in PBS.

For relative quantification of human fibrinogen adsorbed on each membrane, an enzyme-linked immunosorbent assay (ELISA) was performed, with the membrane as the primary substrate. The membranes were incubated in $200 \mu \mathrm{l}$ monoclonal mouse anti-fibrinogen antibody solution (Sigma-Aldrich, Switzerland; 1:1000 in PBS) for $60 \mathrm{~min}$ followed by the same washing procedure. Subsequently, samples were covered with anti-mouse horseradish peroxidase antibodyconjugate [EnVision+Kit (HRP.Mouse); Dako, Switzerland] solution for $30 \mathrm{~min}$. After washing in $500 \mu \mathrm{l}$ PBS three times for $5 \mathrm{~min}$ each and transferring into new wells, $200 \mu \mathrm{l}$ of $3,3^{\prime}, 5,5^{\prime}$-tetramethylbenzidine substrate solution (highest sensitivity; Abcam, UK) was added. The reaction was stopped by adding an equal amount of $450 \mathrm{~nm}$ stop solution (Abcam, UK), and $100 \mu \mathrm{l}$ of the supernatant was transferred into 96-well plate in triplicates per group and measured at $450 \mathrm{~nm}$ excitation wavelength (Mithras2 Plate reader, Berthold Technologies, Germany). Three independent experiments with three replicates each were performed.

\section{F. In vitro cytocompatibility}

All cell culture experiments were performed according to a previously developed protocol. ${ }^{24}$ Briefly, electrospun membranes of $6 \mathrm{~mm}$ in diameter were prepared as described in Sec. II E. Membrane-containing well plates were sterilized by UV irradiation for $4 \mathrm{~h}$. HUVECs (CRL-1730, ATCC, USA) were expanded and used up to passage 18 on gelatin-coated culture flasks in HAM F12-K culture medium (Life Technologies, Thermo Fisher Scientific, Switzerland), supplemented with $10 \%$ fetal calf serum (FCS; Life Technologies, Thermo Fisher Scientific, Switzerland), $1 \%(\mathrm{v} / \mathrm{v})$ penicillin/streptomycin/ neomycin, $1 \%$ L-glutamine, $0.03 \mathrm{mg} \mathrm{ml}^{-1}$ endothelial cell growth supplement (Sigma-Aldrich, Switzerland), and $17 \mathrm{U} \mathrm{ml}^{-1}$ heparin (Sigma-Aldrich, Switzerland). HUVECs were seeded at a density of 25000 cells per membrane in $50 \mu \mathrm{l}$ medium and were allowed to adhere for $2 \mathrm{~h}$ before adding $500 \mu \mathrm{l}$ medium. HUVECs were cultured for 7 days with media change every $48 \mathrm{~h}$.

Cell metabolic activity was assessed on days 1, 3, and 7 postseeding by a Prestoblue assay (Life Technologies, Thermo Fisher Scientific, Switzerland). Cells were incubated 
for $20 \mathrm{~min}$ at $37^{\circ} \mathrm{C}$ in $10 \%$ (v/v) Prestoblue in standard cell culture medium. Prestoblue reduction was quantified based on fluorescent excitation and emission at $\lambda_{\mathrm{ex}}=547 \mathrm{~nm}$ and $\lambda_{\mathrm{em}}=582 \mathrm{~nm}$ (Mithras2 Plate reader, Berthold Technologies, Germany).

Cell growth was assessed based on DNA quantification with the CyQuant assay (Sigma-Aldrich, Switzerland) according to the manufacturer's protocol. Fluorescence intensity was quantified at $\lambda_{\mathrm{ex}}=480 \mathrm{~nm}$ and $\lambda_{\mathrm{em}}=520 \mathrm{~nm}$.

Cell morphology was investigated by SEM. Briefly, cells were fixed in modified Karnovsky fixing solution for $1 \mathrm{~h}$, dehydrated in an ascending ethanol series $(30 \%, 50 \%, 70 \%$, $80 \%, 90 \%$, and $100 \%$ ), and dried in hexamethyldisilazane (Sigma-Aldrich, Switzerland) for $5 \mathrm{~min}$. After overnight drying, membranes were sputter coated and SEM micrographs were acquired as described in Sec. II D.

For fluorescence imaging with a Confocal Laser Scanning Microscopy (CLSM, LSM780, Carl Zeiss AG, Switzerland), cells were fixed in $4 \%$ (w/v) paraformaldehyde (PFA, Sigma-Aldrich, Switzerland) and $0.1 \%(\mathrm{v} / \mathrm{v})$ Triton-X 100 (Sigma-Aldrich, Switzerland). Samples were blocked for unspecific binding in $4 \%(\mathrm{w} / \mathrm{v})$ BSA/PBS. Actin was stained with Alexa-488 conjugated phalloidin [1:200 in 4\% (w/v) BSA/PBS] Life Technologies, Thermo Fisher Scientific, Switzerland), and nuclei were counterstained with 4',6-diamidino-2-phenylindole [1:1000 in 4\% (w/v) BSA/PBS; Life Technologies, Thermo Fisher Scientific, Switzerland]. Three independent experiments with three replicates each were performed for each assay and time points.

\section{G. Blood-material interaction}

Electrospun membranes of $20 \mathrm{~mm}$ in diameter were prewetted in $70 \%$ ethanol solution and incubated in $1 \mathrm{ml}$ PBS overnight. Prior to blood incubation, they were placed into a custom-built PTFE chamber. Human whole blood was freshly taken from healthy volunteers (ethical approval BASEC No. PB_2016-00816 from the local ethics committee, St. Gallen, Switzerland) using the standard venipuncture technique, partially heparinized $\left(0.43 \mathrm{IU} \mathrm{ml}^{-1}\right.$; Carl Roth, Germany) and used within $1 \mathrm{~h}$ after withdrawal. The PTFE chambers were filled with $2.8 \mathrm{ml}$ of human whole blood supplemented with Alexa Fluor 488-conjugated fibrinogen $\left(18 \mu \mathrm{g} \mathrm{ml}^{-1}\right)$ (Thermo Fisher Scientific, Switzerland), closed with a PTFE lid, sealed with parafilm and placed on an orbital shaker (Polymax, Heidolph, Germany) at $10 \mathrm{rpm}$ and RT. After $1 \mathrm{~h}$ of incubation, the blood was collected and stabilized with ethylene-diamine-tetra-acetic acid (Sigma-Aldrich, Switzerland) with a final concentration of $5 \mathrm{mM}$. Blood plasma was separated from stabilized blood samples by centrifugation for $10 \mathrm{~min}$ at $3000 \mathrm{~g}$ and stored at $-80^{\circ} \mathrm{C}$ until further analysis. Levels of platelet factor 4 (PF4) in the supernatant were measured via PF4 Human ELISA kit (Thermo Fisher Scientific, Switzerland). Three independent experiments (accounting for three different donors) were performed with two replicates each. After blood incubation, the scaffolds were rinsed with PBS. One sample of each group was fixed, dehydrated, and sputter coated (10 $\mathrm{nm}$ of thickness) prior to SEM imaging as described in Sec. II F. The second sample was fixed in an aqueous solution containing $4 \%$ (w/v) PFA, $65 \mathrm{mM}$ piperazine- $N, N$-bis(2-ethanesulfonic-acid), $25 \mathrm{mM}$ 4-(2-hydroxyethyl)-1-piperazine-ethanesulfonic-acid, $10 \mathrm{mM}$ ethylene glycol-bis( $\beta$-aminoethyl ether)- $N, N, N^{\prime}, N^{\prime}$-tetra-acetic acid, and $3 \mathrm{mM} \mathrm{MgCl} 2$ (all from Sigma-Aldrich) for $60 \mathrm{~min}$ for immunofluorescence staining and CLSM imaging. After washing with PBS, samples were blocked with 5\% goat serum and $1 \%$ FCS in PBS for $1 \mathrm{~h}$. Cells were permeabilized for $10 \mathrm{~min}$ in PBS containing $0.1 \%$ (v/v) Triton-X 100 (Sigma-Aldrich, Switzerland) and actin was stained with Alexa Fluor 546-conjugated phalloidin [1:200 in 1\% (v/v) FCS/PBS, Thermo Fisher Scientific, Switzerland] for $1 \mathrm{~h}$. All incubation steps were followed by washing in PBS.

\section{H. Statistical analysis}

All data are expressed as mean \pm standard deviation (SD). Mann-Whitney Rank Sum Test was employed to compare the groups using GRAPHPAD PRISM6 software. A value of $p<0.05$ was set as significance level.

\section{RESULTS}

\section{A. HSP allows prediction of solvent mixtures for PCL electrospinning}

The solubility sphere of PCL was constructed with its center coordinate values $\left(\delta D=17.7 \mathrm{MPa}^{1 / 2}, \delta P=5.0 \mathrm{MPa}^{1 / 2}\right.$, and $\left.\delta H=8.4 \mathrm{MPa}^{1 / 2}\right)$ and interaction radius $(R=8)$ value taken from the HSPIP software. As depicted in Fig. 1(a), $\mathrm{CHCl}_{3}, \mathrm{CHCl}_{3}$ :DMF, $\mathrm{CHCl}_{3}$ :DMSO, $\mathrm{CHCl}_{3}$ :acetonitrile, and $\mathrm{CHCl}_{3}$ :methanol mixtures were all located within the PCL solubility sphere with initial RED values of 0.41 , $0.28,0.29,0.34$, and 0.18, respectively. DMF (1.15), DMSO (1.45), acetonitrile (1.76), or methanol (2.1) was outside of the sphere indicating their behavior as a nonsolvent. Subsequently, the evaporation of these solvent:nonsolvent mixtures from PCL solutions was calculated to simulate the jet evolution during fiber formation. $\mathrm{CHCl}_{3}$ evaporation from the solvent mixtures of $\mathrm{CHCl}_{3}$ :methanol and $\mathrm{CHCl}_{3}$ : acetonitrile occurred within a timeframe of 30 a.u. whereas from $\mathrm{CHCl}_{3}$ :DMF and $\mathrm{CHCl}_{3}$ :DMSO solutions within 36.7 a.u. [Fig. S1(a)]. ${ }^{55}$ Moreover, methanol, acetonitrile, or chloroform evaporated within a comparable timeframe, whereas both DMF and DMSO required a longer time for evaporation [Fig. S1(b)]. ${ }^{55}$

$T_{\mathrm{dp}}$ at $35 \% \mathrm{RH}$ and $20 \pm 0.1^{\circ} \mathrm{C}$ RT was $4.1^{\circ} \mathrm{C}$, and $T_{\mathrm{wb}}$ of $\mathrm{CHCl}_{3}$ :DMF and $\mathrm{CHCl}_{3}$ :DMSO solutions was $3.5^{\circ} \mathrm{C}$ and initially below this $T_{\mathrm{dp}}$. Therefore, for both solutions, $\Delta T$ was higher than zero leading to water condensation on the jet surface. After 26.7 a.u., $T_{\text {wb }}$ of both solutions rapidly increased, reaching values higher than $T_{\mathrm{dp}}$, resulting in ceasing of water condensation [Fig. 1(b)]. The RED value of $\mathrm{CHCl}_{3}$ :DMSO reached 1.0 earlier than the one of $\mathrm{CHCl}_{3}$ :DMF [Fig. 1(c)]. 

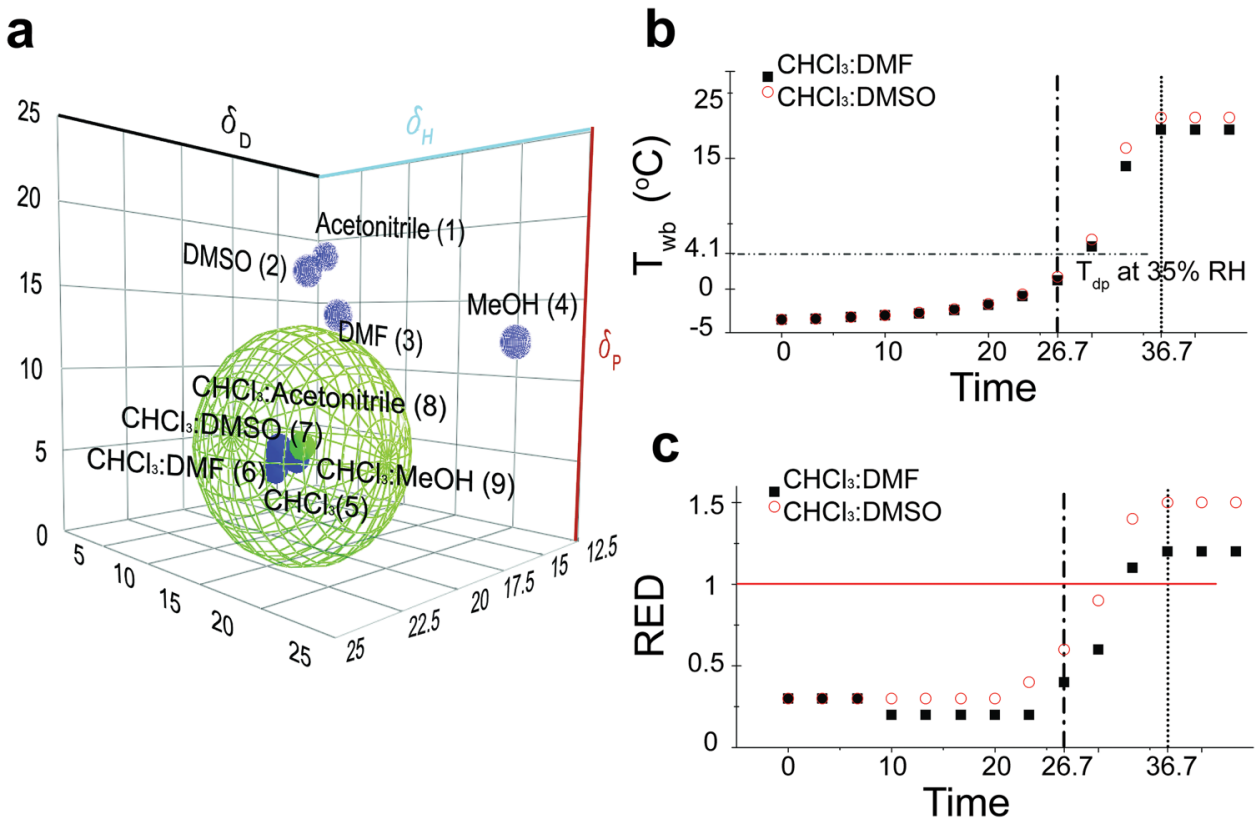

FIG. 1. (a) 3D Hansen space displaying PCL solubility sphere (green dot represents its center) and solvents: Nonsolvents for PCL, acetonitrile (1), DMSO (2), DMF (3), and $\mathrm{MeOH}$ (4) are located outside of this PCL solubility sphere, whereas good solvents/solvent mixtures for PCL, $\mathrm{CHCl}_{3}(5), \mathrm{CHCl}_{3}: \mathrm{DMF}$ (6), $\mathrm{CHCl}_{3}$ :DMSO (7), $\mathrm{CHCl}_{3}$ :acetonitrile (8), and $\mathrm{CHCl}_{3}: \mathrm{MeOH}$ (9) are inside; (b) $T_{\mathrm{dp}}$ of electrospinning environment at $35 \% \mathrm{RH}$ is $4.1{ }^{\circ} \mathrm{C}$ and this (dasheddotted-dotted line) is marked. $T_{\mathrm{wb}}$ changes $\left({ }^{\circ} \mathrm{C}\right)$ during solvent evaporation from $\mathrm{CHCl}_{3}$ :DMF and $\mathrm{CHCl}_{3}$ :DMSO solutions were simulated; (c) $\mathrm{RED}$ value changes were calculated during solvent evaporation from $\mathrm{CHCl}_{3}: \mathrm{DMF}$ and $\mathrm{CHCl}_{3}: \mathrm{DMSO}$ solutions; (dashed-dotted line) last time point of similar amounts of solvent (S) and nonsolvent; (round dotted line) time point $S=0$; (red line) solubility limit for the polymer in the solvent system.

\section{B. Development of PCL electrospun fibers with controlled diameter and tailored surface structures}

As predicted by the HSP model, electrospinning of two different PCL solutions prepared with $\mathrm{CHCl}_{3}$ :DMF or $\mathrm{CHCl}_{3}$ : DMSO yielded micron-sized fibers with two distinct surface topographies as shown in Fig. 2(a). When PCL dissolved in $\mathrm{CHCl}_{3}$ :DMSO was electrospun, highly structured microfibers (MF) were obtained in contrast to MF electrospun from $\mathrm{CHCl}_{3}$ :DMF at the same environmental conditions. Despite the different surface morphology, fibers exhibited comparable diameters of $3.7 \pm 0.3 \mu \mathrm{m}$ and $3.6 \pm 0.4 \mu \mathrm{m}$ for $\mathrm{MF}$ and structured MF, respectively [Fig. 2(b)].

The different surface topography was reflected with significantly higher specific surface area (SSA) for structured MF samples with $2.32 \pm 0.49 \mathrm{~m}^{2} \mathrm{~g}^{-1}$ as opposed to $0.76 \pm$ $0.23 \mathrm{~m}^{2} \mathrm{~g}^{-1}$ for MF calculated from $\mathrm{Kr}$ adsorption isotherms as shown in Fig. 3(c). The macroscopic roughness, on the other hand, was similar for both membranes with $S_{q}$ values of $13.5 \pm 1.4 \mu \mathrm{m}$ for MF and 14.2 $\pm 1.5 \mu \mathrm{m}$ for structured MF [Fig. 3(a)]. Furthermore, comparable water contact angles
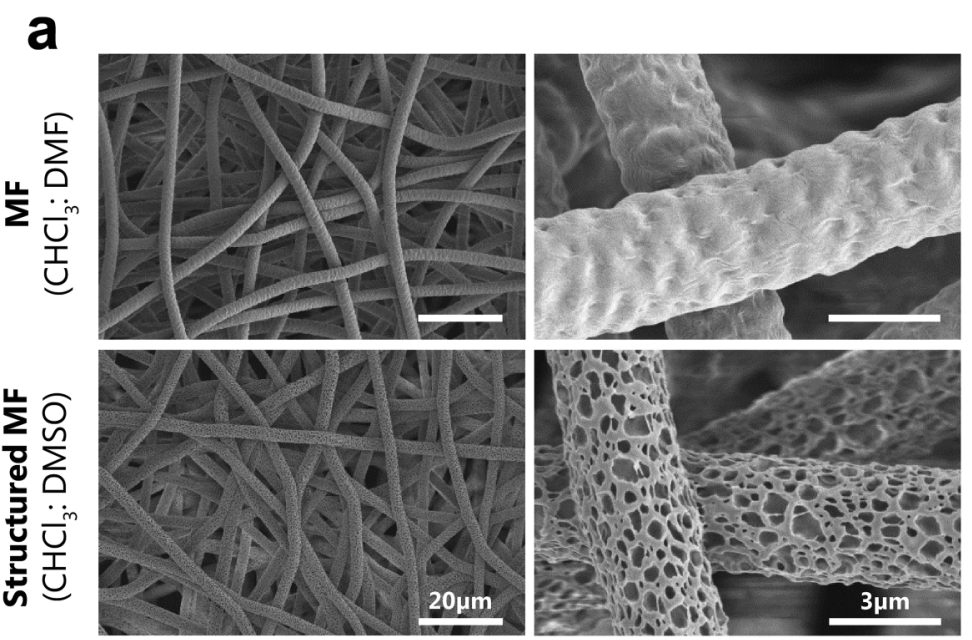

b

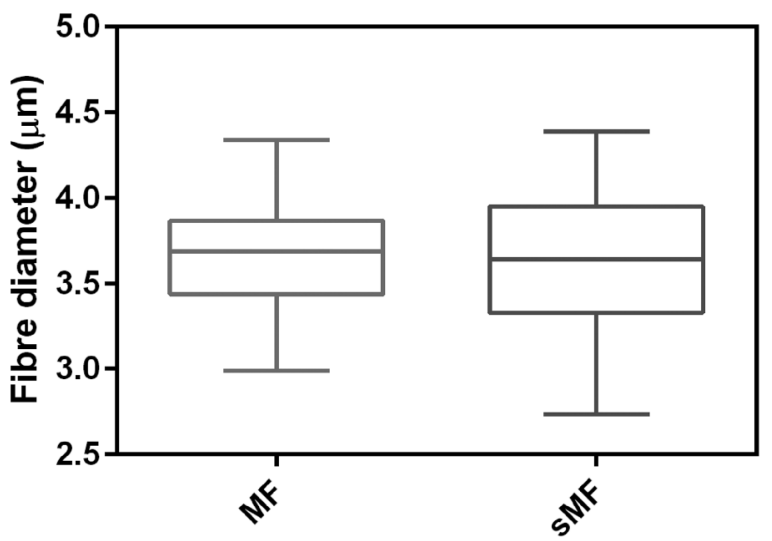

FIG. 2. (a) SEM micrographs of $\mathrm{MF}\left(\mathrm{CHCl}_{3}: \mathrm{DMF}\right)$ and structured $\mathrm{MF}\left(\mathrm{CHCl}_{3}: \mathrm{DMSO}\right)$ in low and high magnification, respectively. (b) Fiber diameter distribution of MF and structured MF (sMF). 
a

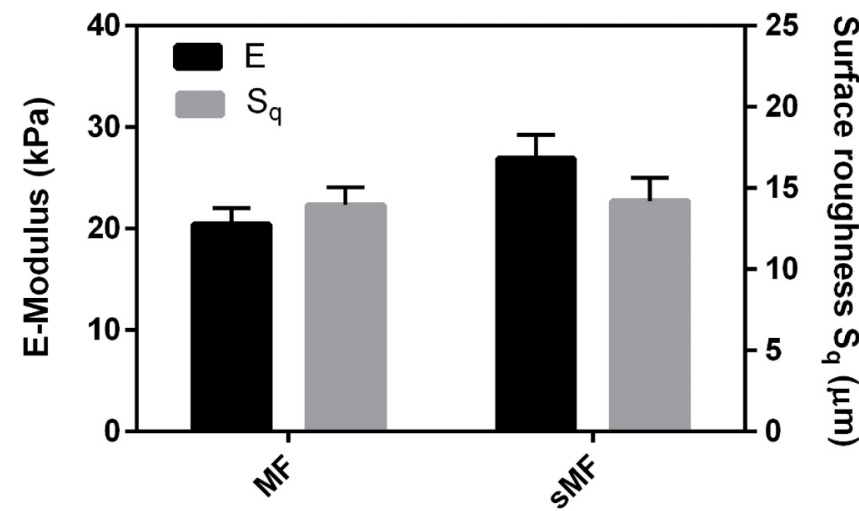

C

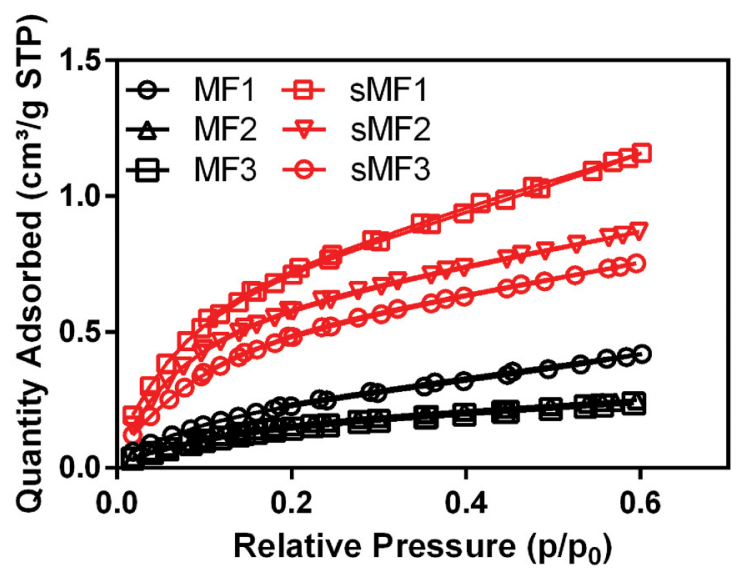

b

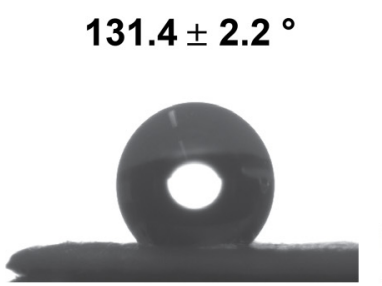

MF
$132.1 \pm 2.2^{\circ}$

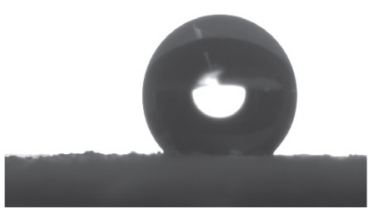

SMF

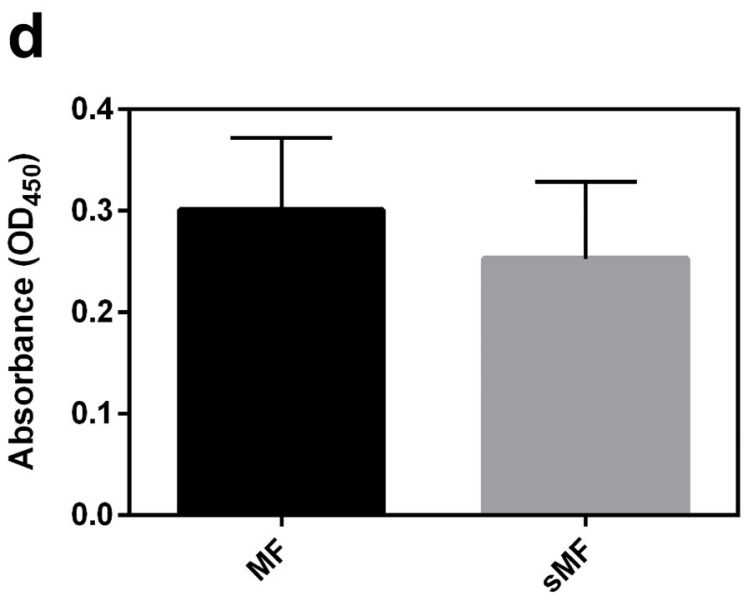

FIG. 3. (a) Elastic moduli of MF and structured MF (sMF) obtained from tensile testing and macroscopic surface roughness $\left(S_{q}\right)$ measured with a confocal $3 \mathrm{D}$ optical surface metrology system, (b) static water contact angle analyzed based on photographs taken with optical contact meter, (c) krypton adsorption and desorption isotherms to calculate SSA by BET, and (d) protein adsorption analyzed via an ELISA based approach. (Results are presented as mean \pm SD of $n=3$.)

were observed for membranes of MF or structured MF with $131.4 \pm 2.2^{\circ}$ or $132.1 \pm 2.2^{\circ}$, respectively [Fig. 3(b)]. Both membranes demonstrated ductile behavior measured by tensile testing with a Young's modulus of $26.9 \pm 1.9 \mathrm{kPa}$ for structured MF and $20.4 \pm 1.3 \mathrm{kPa}$ for MF [Fig. 3(a)]. Moreover, the relative amount of fibrinogen adsorption on the electrospun membranes was not statistically different for MF $(0.300 \pm 0.071$ a.u. $)$ compared to structured MF membranes $(0.253 \pm 0.075$ a.u. $)$ [Fig. $3(d)]$.

\section{MF and structured MF are cytocompatible independent of fiber surface structure}

To evaluate the cytocompatibility of the PCL membranes, the metabolic activity, increase in amount of DNA, and morphology of HUVECs cultured on MF and structured MF membranes were assessed 1, 3, and 7 days postseeding.

On both membranes, HUVECs adhered well and formed a homogeneous monolayer of cells over time [Fig. 4(a)]. Cells proliferated on the scaffolds, increasing significantly in metabolic activity and DNA amount within the culture period [Figs. 4(b) and 4(c)]. No significant differences with respect to DNA amount or metabolic activity were observed between cells on MF and structured MF membranes, except on day 7. The amount of DNA from cells on structured MF showed a small but significant increase when compared to cells cultured on MF.

\section{Blood-material interaction with electrospun membranes}

After membrane incubation in human whole blood, platelet activation was assessed by measuring the release of PF4 by ELISA in the supernatant. The results exhibited comparable levels of platelet activation on MF and structured MF [Fig. 5(b)], although with high variations between experiments due to donor-to-donor variability. Neither of the membrane types induced strong coagulation but in accordance with PF4 levels, comparable areas of fibrin network formation were observed on MF and structured MF, as indicated in SEM micrographs [Fig. 5(a)].

Visualizing the fibrin network and attachment of platelets by CLSM imaging additionally demonstrated that MF membranes caused apparent comparable degrees of fibrin network formation and platelet attachment independent of the fiber surface structure.

\section{DISCUSSION}

It is known that EC and blood behavior can be steered by changing substrate characteristics such as surface roughness, ${ }^{11,17}$ 
a
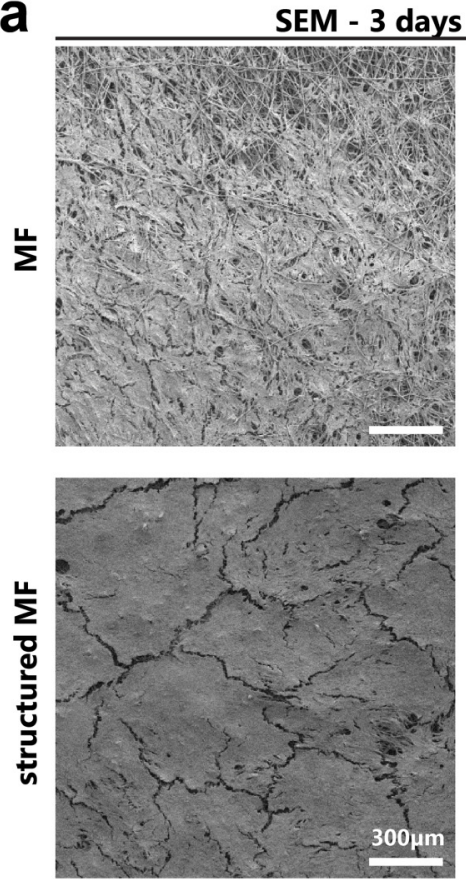

b

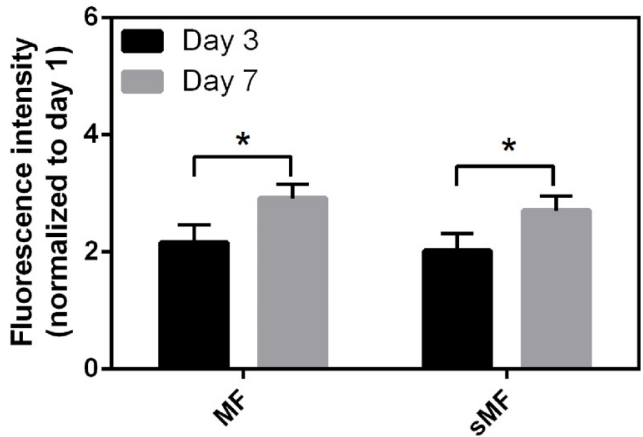

Immunofluorescence
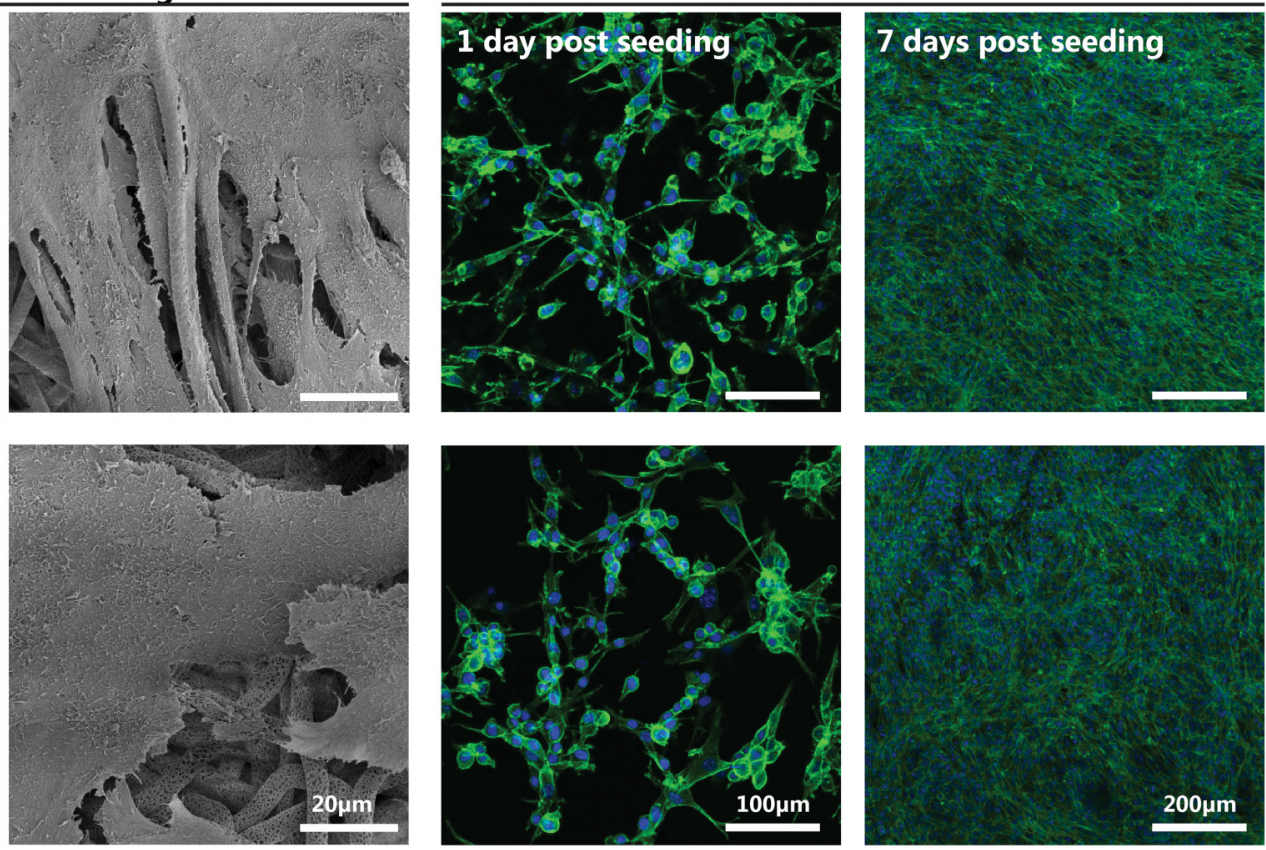

C

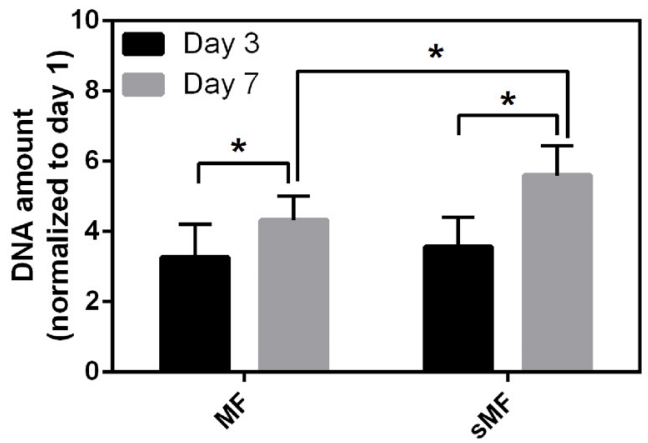

FIG. 4. (a) SEM micrographs of HUVECs on MF and structured MF 3 days postseeding. CLSM images of immunofluorescence staining of cytoskeletal actin (green) and cell nucleus (blue) of HUVECs on MF and structured MF on day 7. CLSM images are maximum intensity projections of z-stacks. (b) Metabolic activity of HUVECs cultured on MF and structured MF (sMF) over 7 days, (c) DNA amount on both membrane types for 7 days. *indicates statistically significant differences with $p<0.05$. (Results are presented as mean $\pm \mathrm{SD}$ of $n=3$ independent experiments with three replicates each.)

stiffness, ${ }^{25-27}$ and wettability. ${ }^{10,28}$ In order to evaluate the influence of fiber surface structure, electrospun membranes were fabricated possessing similar fiber diameters and thus surface roughness, water contact angles, and mechanical properties while differing only in their fiber surface morphology.

To this end, a theoretical model was used in order to predict solvent systems for PCL, which leads to electrospun fibers with the aforementioned desired characteristics of similar fiber diameter yet different fiber surface structure. Based on the simulation, information from solvent evaporation as well as subsequent $T_{\mathrm{wb}}$ and RED changes, which are the driving forces leading to electrospun fiber surface structure formation, was combined.

It was previously reported that PCL solutions electrospun from $\mathrm{CHCl}_{3}$ :DMSO solvent mixture at high $\mathrm{RH}$ conditions yield fibers with structured surfaces. The fiber diameter can be greatly affected by the solvent mixture and chosen spinning conditions. Among our solvent mixtures evaluated for PCL,
$\mathrm{CHCl}_{3}$ :DMF was the closest one to $\mathrm{CHCl}_{3}$ :DMSO in terms of solvent evaporation due to close water vapor pressure values of DMF or DMSO (3.8 and $0.7 \mathrm{~mm} \mathrm{Hg}$ at $21^{\circ} \mathrm{C}$, respectively) compared to $\mathrm{MeOH}(103 \mathrm{~mm} \mathrm{Hg})$ or acetonitrile $(71 \mathrm{~mm}$ $\mathrm{Hg}){ }^{29}$ Additionally, dielectric constants of DMF (36.7) and DMSO (46.6) are in a similar range. ${ }^{29}$ Therefore, $\mathrm{CHCl}_{3}$ : DMF and $\mathrm{CHCl}_{3}$ :DMSO solvent mixtures were predicted to yield fibers with comparable diameters. This was experimentally confirmed by SEM imaging of fibers spun from all four solvent systems as shown in Fig. S2. ${ }^{55}$ To further elaborate on the surface structure on fibers electrospun from $\mathrm{CHCl}_{3}$ :DMF and $\mathrm{CHCl}_{3}$ :DMSO, solvent mixtures were evaluated in terms of (i) surface temperature change that defines the water condensation on the spinning jet as well as (ii) RED change, which indicates the phase separation. The solvent mixture $\mathrm{CHCl}_{3}$ :DMSO is reaching an RED value of 1.0 at an earlier timepoint than the one of $\mathrm{CHCl}_{3}$ :DMF. This indicates that a $\mathrm{CHCl}_{3}$ :DMSO solution is more prone to phase separation 

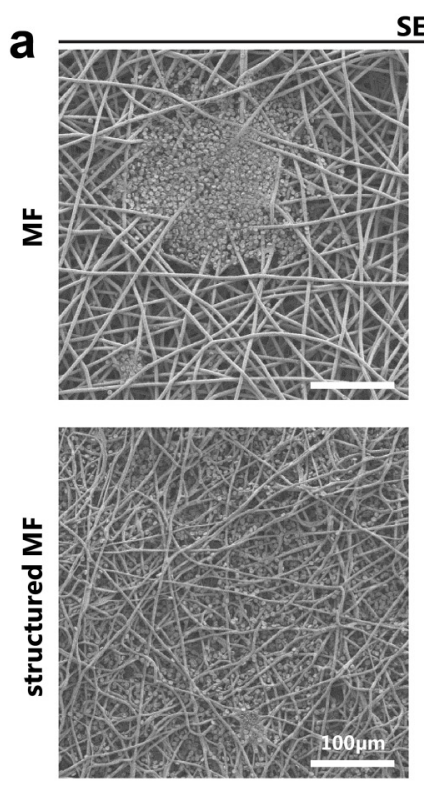

SEM
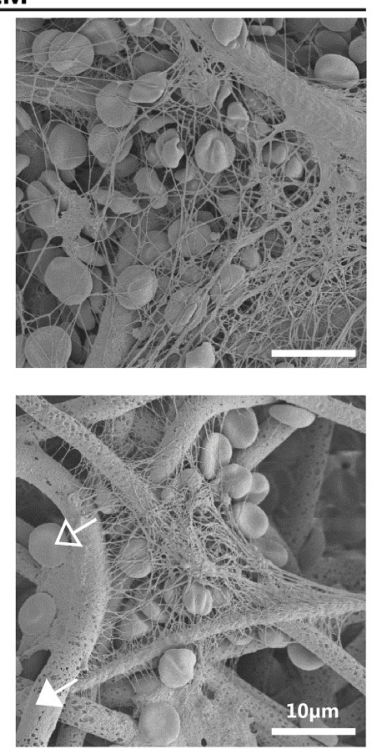
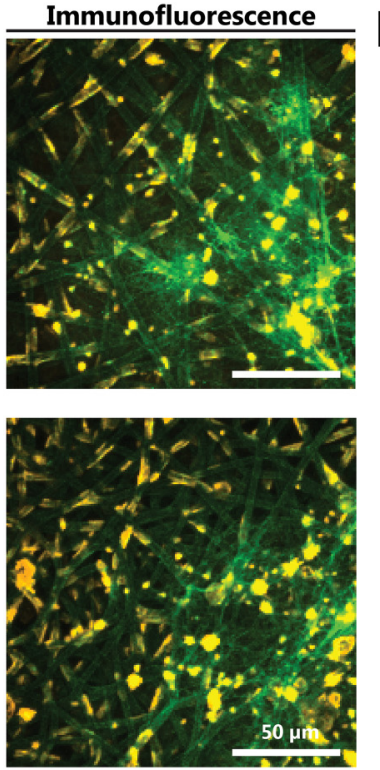

b

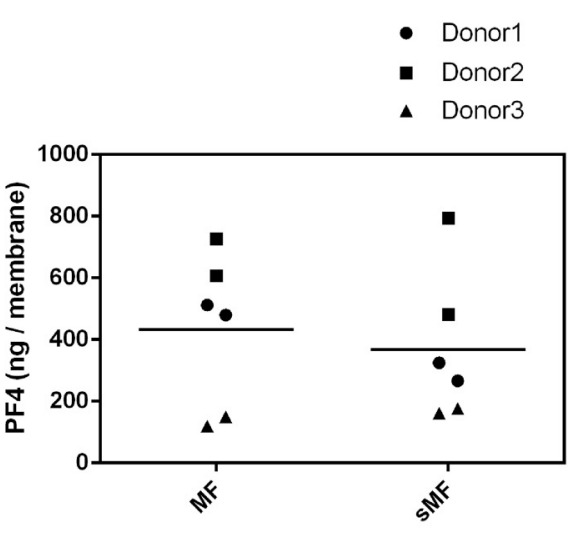

FIG. 5. (a) SEM micrographs show fibrin network on MF and structured MF. CLSM imaging of immunofluorescence staining of platelet cytoskeletal actin (orange) and fibrinogen in fibrin networks (green) shown after $1 \mathrm{~h}$ whole blood incubation on MF and structured MF. Images are maximum intensity projections of z-stacks. (b) Concentrations of PF4 in the supernatants after incubation of the membranes in human whole blood. ( $n=3$ donors, assessed in three independent experiments with two replicates each. Empty arrow indicates disclike red blood cells, and filled arrow indicates platelets.)

accounting also for the water condensation at the given RH. The water condensation can therefore enhance the increase in RED even further for the herewith investigated PCL in $\mathrm{CHCl}_{3}$ :DMSO solution, since water is a nonsolvent for the hydrophobic polymer PCL. According to this evaluation, this would yield more pronounced structures on the electrospun fiber surface compared to $\mathrm{CHCl}_{3}$ :DMF solution. ${ }^{16}$ Both theoretical predictions of comparable fiber diameter and distinct surface structure were seconded by experimental evidence and confirmed by SEM micrographs.

With a similar approach, Casasola et al. used initial RED values of solvent-polymer pairs, calculated with HSP, to preselect solvents for poly(lactic acid) dissolution for electrospinning. ${ }^{30}$ In another study by Luo et al., binary solvent mixtures of poly(methylsilsesquioxane) were chosen based on predictions with a Teas graph. Teas graph is a 2D empirical solubility parameter approach derived from HSP. They showed that the differences in solvent volatility or polymer solubility of the solvent pairs could initiate surface structure on electrospun fibers. ${ }^{31}$ Our prediction thus agrees with these approaches as well as with the findings of our previous study, ${ }^{16}$ which revealed that the interplay between the polymer solubility changes (RED), jet surface temperature $\left(T_{\mathrm{wb}}\right)$, and their synergistic effect, together with applied RH shaped the surface structuring on electrospun fibers.

The solvent systems incorporating DMF or DMSO in chloroform showed best comparability in terms of fiber diameters but most pronounced differences in surface texturing. As displayed in Fig. 2, even smooth fibers created with $\mathrm{CHCl}_{3}: \mathrm{DMF}$ show a slightly textured surface, which is, however, not comparable to the significant structures and holes observed in the structured fibers. They were thus chosen for the investigation of cell-surface and blood-surface interactions.
It is known that solvent systems also have an influence on the mechanical properties of resulting electrospun fibers. ${ }^{32-34}$ However, these effects are solvent and material specific. Since our results of stress-strain evaluation suggest very comparable mechanical properties as shown by similar Young's moduli of the membrane types, the effect of $\mathrm{CHCl}_{3}$ as the main solvent component probably outweighs the effects from the small portion $(10 \%)$ of the other solvents.

Similarly to Milleret et al., ${ }^{11}$ overall surface roughness of MF or structured MF values of electrospun membranes were calculated using an image based method. The presented values reflect the comparable fiber diameter and spacing as well as a similar fiber assembly during electrospinning. During imaging, steep surfaces with slopes $>42^{\circ}$ from fiber edges and deep buried fibers gave weak signal intensity and thus lead to a resolution limit that does not allow for nanoscale fiber surface topography to be measured. With appropriate adjustments, images of electrospun membranes could be acquired (Fig. S3), ${ }^{55}$ and surface roughness of $S_{q, \mathrm{MF}}=13.5 \pm 1.4 \mu \mathrm{m}$ and $S_{q, \mathrm{sMF}}=14.2 \pm 1.5 \mu \mathrm{m}$ could be calculated to give a good overall impression of the macroscopic surface topography.

It has been shown that fiber diameter of electrospun membranes can steer HUVEC attachment and morphology. ${ }^{12}$ Besides, not only fiber diameter, but also nanosized fiber surface structure were found to enhance the proliferation and spreading of smooth vascular cells and mesenchymal stem cells, ${ }^{15,35}$ to improve the functionality of hepatocytes, ${ }^{36}$ and to reduce the spreading of macrophages without changing metabolic activity or cell number. ${ }^{37}$ Contrasting these effects of fiber surface structure on various cell types, results provided in this study demonstrate comparable HUVEC behavior on MF and structured MF membranes. 
Zhou et al. suggested that the enhanced attachment and proliferation of SMCs is the result of increased protein adsorption due to enlarged fiber surface structure, higher hydrophobicity, and specific surface area on aligned PLLA fibers. ${ }^{15}$ In contrast, in this study, PCL membranes were produced with randomly deposited fibers, which result in generally higher contact angles. ${ }^{38,39}$ The similar wettability of PCL MF and structured MF membranes is likely the result of comparable macroscopic roughness and prominent interfiber porosity, which outweighed the effect of fiber surface structure. This is in agreement with Ma et al. reporting contact angles of 144 and $147^{\circ} \mathrm{C}$ for poly(methyl methacrylate) electrospun membranes with smooth or structured microfibers, respectively. ${ }^{40}$ The insensitivity of HUVECs to structures on the fiber surface might therefore be caused by similar water contact angles and amount of protein adsorption on MF and structured MF membranes.

It is known that cellular interaction with materials is cell type specific. ${ }^{41,42}$ It has even been reported that ECs show a cell origin dependent responsiveness to substrates. ${ }^{43}$ To investigate if primary cells are more sensitive to structured fibers, HUVECs from a source of pooled isolated primary cells were cultured on MF or structured MF membranes (Fig. S5). ${ }^{55}$ Even though these cells showed decreased metabolic activity over the culture period and a low degree of spreading, their response was independent of fiber morphology (MF versus structured MF). The impaired viability of these cells on the fiber membranes could potentially be improved by additional surface modification via biofunctionalization by vascular endothelial growth factor or cell adhesive ligand immobilization. ${ }^{44-46}$

It has been shown previously that platelet adhesion and thrombus formation can be suppressed by the addition of surface topographical features in the range of $50 \mathrm{~nm}$ to $2 \mu \mathrm{m}$ due to reduced platelet-surface contact..$^{8,47,48}$ In the case of 3D scaffolds, electrospun membranes with fibers smaller than $1 \mu \mathrm{m}$ in diameter have been shown to induce a lower coagulation activation cascade and less platelet adhesion in comparison to scaffolds composed of thicker fibers. ${ }^{11}$ Interestingly, the current study shows that the herewith presented micron-sized fibers with surface structures in the range of $100 \mathrm{~nm}$ to $1 \mu \mathrm{m}$ have no measurable effect on platelet activation and thus fibrin network formation. Fibrinogen is a key protein of platelet adhesion and activation. ${ }^{49}$ The comparable amount of fibrinogen adsorbed on MF and structured MF already suggests that coagulation behavior is likely to be similar for these fiber meshes.

Based on the literature and previously published experimental evidence, the presented research was driven by the rationale that nanostructures on micron-sized electrospun fibers would (a) reduce platelet adhesion to provide an anticoagulant surface and (b) substantially influence attachment and proliferation of HUVECs. To this end, a system was established where only the fiber surface structure is the discriminating factor between the membranes. However, with the membranes being similar in all other aspects, i.e., water contact angle, macroscopic roughness, and protein adsorption, the herewith presented experimental design could not detect such difference in the response of HUVECs and blood to the fiber membranes. The results thus suggest that both structured and nonstructured MF membranes are suitable substrates for vascular tissue engineering. Of note, results on blood-material interaction are greatly donor dependent, and the inherent variability might obscure potential differences between materials. Quantification of platelet attachment and activation, however, necessitates further investigations, including the development of highly sensitive methods to elucidate more subtle changes in blood coagulation parameters and platelet activation. Our results draw attention to the continuing complexity and difficulty when interpreting results in biology-material interaction studies. Thereby, this work contributes toward the understanding of HUVEC and bloodmaterial interaction on electrospun membranes. Furthermore such structured fibers offer additional interesting avenues for vascular tissue engineering applications: larger surface area provides not only enhanced loading capacity for biofunctional molecules but also accelerates the degradation of polyesters and increases the drug release. ${ }^{50,51}$ Hypothetically, electrospun membranes with fiber surface structures could be exploited to match the degradation rate of scaffolds with tissue regeneration $^{52}$ and to tune drug release for enhanced early endothelialization $^{53}$ in small diameter vascular graft applications. This holds true also for aforementioned protein or growth factor immobilization, where larger surface area can contribute to increased loading capacity. ${ }^{54}$

\section{SUMMARY AND CONCLUSIONS}

In summary, by using a systematic prediction approach based on HSP, two types of electrospun membranes possessing a similar fiber diameter in the micrometer range with one exhibiting fiber surface structures in the submicrometer range were developed. Through in vitro assessment of HUVECs and human whole blood interaction with the generated membranes, it was observed that structured microfibers induced similar platelet activation and coagulation as nonstructured fibers while also showing similar endothelialization potential. Hence, the introduction of the fiber surface structure on electrospun membranes cannot be used to mitigate the inferior blood coagulation performance associated with micron-sized fiber membranes, but can potentially be used for further chemical functionalization, drug release, or tuning of degradation behavior in order to ultimately achieve a compliant vascular graft.

\section{ACKNOWLEDGMENTS}

The authors thankfully acknowledge the support on electrospinning the fiber membranes by Vasundhara Tyagi, Empa. This work is part of the Zurich Heart project under the umbrella of University Medicine Zurich/Hochschulmedizin Zürich.

${ }^{1}$ G. A. Roth, M. H. Forouzanfar, A. E. Moran, R. Barber, G. Nguyen, V. L. Feigin, M. Naghavi, G. A. Mensah, and C. J. L. Murray, N. Engl. J. Med. 372, 1333 (2015). 
${ }^{2}$ S. Pashneh-Tala, S. MacNeil, and F. Claeyssens, Tissue Eng. Part B Rev. 22, 68 (2015).

${ }^{3}$ L. Gui, A. Muto, S. A. Chan, C. K. Breuer, and L. E. Niklason, Tissue Eng. Part A 15, 2665 (2009).

${ }^{4}$ A. Hasan, A. Memic, N. Annabi, M. Hossain, A. Paul, M. R. Dokmeci, F. Dehghani, and A. Khademhosseini, Acta Biomater. 10, 11 (2014).

${ }^{5}$ F. Jung and S. Braune, Biointerphases 11, 029601 (2016).

${ }^{6}$ E. Potthoff, D. Franco, and V. D’Alessandro, Nano Lett. 14, 1069 (2014).

${ }^{7}$ P. Formentín, Ú Catalán, S. Fernández-Castillejo, M. Alba, M. Baranowska, R. Solà, J. Pallarès, and L. F. Marsal, J. Biomater. Appl. 30, 1 (2015).

${ }^{8}$ L. B. Koh, I. Rodriguez, and S. S. Venkatraman, Biomaterials 31, 1533 (2010).

${ }^{9}$ M. Hulander, A. Lundgren, L. Faxälv, T. L. Lindahl, A. Palmquist, M. Berglin, and H. Elwing, Colloids Surf. B 110, 261 (2013).

${ }^{10}$ S. Movafaghi, V. Leszczak, W. Wang, J. A. Sorkin, L. P. Dasi, K. C. Popat, and A. K. Kota, Adv. Healthc. Mater. 6, 1600717 (2017).

${ }^{11}$ V. Milleret, T. Hefti, H. Hall, V. Vogel, and D. Eberli, Acta Biomater. 8, 4349 (2012).

${ }^{12}$ E. S. Fioretta, M. Simonet, A. I. P. M. Smits, F. P. T. Baaijens, and C. V. C. Bouten, Biomacromolecules 15, 821 (2014).

${ }^{13}$ S. Lamichhane, J. A. Anderson, T. Remund, H. Sun, M. K. Larson, P. Kelly, and G. Mani, J. Biomed. Mater. Res. A 104, 2291 (2016).

${ }^{14}$ F. Ahmed, N. R. Choudhury, N. K. Dutta, S. Brito, A. Zannettino, and E. Duncan, Biomacromolecules 15, 744 (2014).

${ }^{15}$ Q. Zhou, J. Xie, M. Bao, H. Yuan, Z. Ye, X. Lou, and Y. Zhang, J. Mater. Chem. B 3, 4439 (2015).

${ }^{16}$ G. Yazgan et al., Sci. Rep. 7, 158 (2017).

${ }^{17}$ H. Bergmeister, C. Schreiber, C. Grasl, I. Walter, R. Plasenzotti, M. Stoiber, D. Bernhard, and H. Schima, Acta Biomater. 9, 6032 (2013).

${ }^{18}$ Z. Wang et al., Biomaterials 35, 5700 (2014).

${ }^{19}$ X. Ren et al., Chem. Soc. Rev. 44, 5680 (2015).

${ }^{20}$ C. M. Hansen, Hansen Solubility Parameters: A User's Handbook, 2nd ed. (CRC, Boca Raton, FL, 2007).

${ }^{21}$ S. Abbott, C. M. Hansen, and H. Yamamoto, eBook: Hansen Solubility Parameters in Practice - Complete with software, data, and examples, 1st ed. 2008, 2nd ed. 2009, 3rd ed. 2010, 4th ed. 2013, 5th ed. 2015, available from www.hansen-solubility.com.

${ }^{22}$ G. Yazgan, A. M. Popa, R. M. Rossi, K. Maniura-Weber, J. Puigmartí-Luis, D. Crespy, and G. Fortunato, Polymer 66, 268 (2015).

${ }^{23}$ S. Brunauer, P. H. Emmett, and E. Teller, J. Am. Chem. Soc. 60, 309 (1938).

${ }^{24}$ A. G. Guex, G. Fortunato, D. Hegemann, H. T. Tevaearai, and M. N. Giraud, Methods Mol. Biol. 1058, 119 (2013).

${ }^{25}$ M. F. Kee, D. R. Myers, Y. Sakurai, W. A. Lam, and Y. Qiu, PLoS One 10, e0126624 (2015).

${ }^{26}$ Y. Qiu et al., Proc. Natl. Acad. Sci. U.S.A. 111, 14430 (2014).

${ }^{27}$ S. Jalali, M. Tafazzoli-Shadpour, N. Haghighipour, R. Omidvar, and F. Safshekan, Cell Commun. Adhes. 22, 79 (2016).

${ }^{28}$ W. L. Song and J. F. Mano, Soft Matter 9, 2985 (2013).

${ }^{29}$ I. M. Smallwood, Organic Solvent Properties (1996).
${ }^{30}$ R. Casasola, N. L. Thomas, and S. Georgiadou, J. Polym. Sci. Part B Polym. Phys. 54, 1483 (2016).

${ }^{31}$ C. J. Luo, M. Nangrejo, and M. Edirisinghe, Polymer 51, 1654 (2010).

${ }^{32}$ Y. Xu, L. Zou, H. Lu, and T. Kang, RSC Adv. 7, 4000 (2017).

${ }^{33}$ Y. Huang and D. R. Paul, J. Polym. Sci. Part B Polym. Phys. 45, 1390 (2007).

${ }^{34}$ X. Liu, S. G. Baldursdottir, J. Aho, H. Qu, L. P. Christensen, J. Rantanen, and M. Yang, Pharm. Res. 34, 738 (2017).

${ }^{35}$ L. Moroni, R. Licht, J. de Boer, J. R. de Wijn, and C. A. van Blitterswijk, Biomaterials 27, 4911 (2006).

${ }^{36}$ T. Wang, Z.-Q. Feng, M. K. Leach, J. Wu, and Q. Jiang, J. Mater. Chem. B 1, 339 (2013).

${ }^{37}$ N. J. Schaub, T. Britton, R. Rajachar, and R. J. Gilbert, ACS Appl. Mater. Interfaces 5, 10173 (2013).

${ }^{38}$ J. I. Kim, T. I. Hwang, L. E. Aguilar, C. H. Park, and C. S. Kim, Sci. Rep. 6, 1 (2016)

${ }^{39}$ H. Jahani, S. Kaviani, M. Hassanpour-Ezatti, M. Soleimani, Z. Kaviani and Z. Zonoubi, Cell J. 14, 31 (2012), see https://www.ncbi.nlm.nih.gov/ pubmed/23626935

${ }^{40}$ M. Ma, M. Gupta, Z. Li, L. Zhai, K. K. Gleason, R. E. Cohen, M. F. Rubner, and G. C. Rutledge, Adv. Mater. 19, 255 (2007).

${ }^{41}$ F. Y. Teng et al., Bioinorg. Chem. Appl. 2016, 3837679.

${ }^{42}$ A. Hunter, C. W. Archer, P. S. Walker, and G. W. Blunn, Biomaterials 16, 287 (1995).

${ }^{43}$ D. Bouïs, G. A. P. Hospers, C. Meijer, G. Molema, and N. H. Mulder, Angiogenesis 4, 91 (2001).

${ }^{44}$ Y. M. Shin, Y. Bin Lee, S. J. Kim, J. K. Kang, J. C. Park, W. Jang, and H. Shin, Biomacromolecules 13, 2020 (2012).

${ }^{45}$ H. Ghanbari, D. Radenkovic, S. M. Marashi, S. Parsno, N. Roohpour, G. Burriesci, and A. M. Seifalian, Biointerphases 11, 029801 (2016).

${ }^{46}$ Y. Xia, F. Boey, and S. S. Venkatraman, Biointerphases 5, FA32 (2010).

${ }^{47}$ X. Liu, L. Yuan, D. Li, Z. Tang, Y. Wang, G. Chen, H. Chen, and J. L. Brash, J. Mater. Chem. B 2, 5718 (2014).

${ }^{48}$ H. Fan et al., Small 5, 2144 (2009).

${ }^{49}$ L. C. Xu, J. W. Bauer, and C. A. Siedlecki, Colloids Surf. B 124, 49 (2014).

${ }^{50}$ Y. Dong, S. Liao, M. Ngiam, C. K. Chan, and S. Ramakrishna, Tissue Eng. Part B Rev. 15, 333 (2009).

${ }^{51}$ X. Hu, S. Liu, G. Zhou, Y. Huang, Z. Xie, and X. Jing, J. Control. Release 185, 12 (2014).

${ }^{52}$ Y. Pan et al., Sci. Rep. 7, 3615 (2017).

${ }^{53}$ Z. Wang et al., ACS Appl. Mater. Interfaces 9, 19541 (2017).

${ }^{54}$ N. R. Mohamad, N. H. C. Marzuki, N. A. Buang, F. Huyop, and R. A. Wahab, Biotechnol. Biotechnol. Equip. 29, 205 (2015).

${ }^{55}$ See supplementary material at https://doi.org/10.1116/1.5047668 for additional information on HSPiP simulations of evaporations from solvent mixtures, SEM images of electrospun fibres from different solvent:nonsolvent mixtures, surface roughness and mechanical properties of fibre membranes as well as the response of primary HUVECs on the fibre membranes. 